\title{
Personalized Mobile System Application: A Case Study
}

\author{
Sternly K. Simon ${ }^{1}$, Siti Fatimah Abdul Razak ${ }^{2}$, Chin-Poo Lee ${ }^{3}$ \\ Faculty of Information Science and Technology, University Multimedia, Melaka, Malaysia \\ E-mail:.1 sternly_simon@yahoo.com, 2fatimah.razak@mmu.edu.my, ${ }^{3}$ cplee@mmu.edu.my
}

\begin{abstract}
This paper presents an overview of the components, approaches and techniques which are used to build a mobile phoneapplication that uses short messaging service (SMS) text messages to improve interaction, information distribution and communication of stakeholders in a university setting. The proposed application is built upon a multiple compatible mobile phone menu-based subscription management application that is also customizable. Since SMS has the potential to fill significant connectivity and service gaps, this application can provide support for them to become more ubiquitous. Event-based approach towards context-aware personalized notification service is adopted, i.e. user will receive relevant immediate SMS to his/her mobile phone based on his/her subscription for preferred notifications. A trigger enables event management system to send out (semi-) automated personalized notification. Notification services that understand the context within which their users operate, i.e. identity, activity and time are derived based on a set of predetermined rules. This will benefit the stakeholders in terms of getting up-to-date notifications via the delivery system which implements SysMan TellMe V8.04 as the delivery protocol and SMS server.
\end{abstract}

Keywords - mobile systems; short messaging service; pervasive technology

\section{INTRODUCTION}

Nowadays, most higher learning institution or universities provide official websites and online systems dedicated to specific departments or units to fulfil students' information needs. For example, Student Information System, Financial Kiosk, Exam Results System and etc. are among those systems usually implemented in a University setting. These systems requires students to access the systems separately each time. This poses a problem to students to manage and access that information in a timely manner. Even tough the Internet is dominant nowadays, not everyone has access to the Internet all the time to take advantage on the services it has to offer. Normally, the University assumes that every student will take it upon their own responsibility to ensure they are aware of new updates related to them, for example announcements, course materials, exam results and etc. However due to time constraints, most students have problem to keep themselves up-to-date.

In this study, we refer to our University as a case study. Based on the case study, student relies heavily on Multimedia Learning System (MMLS), Integrated Computerised Education Management System (ICEMS), Event Management System and Online Academic Evaluation System to facilitate their studies.

ICEMS is used as a mechanism for course registration to take place. Student can register courses based on the online schedule provided. However, in many student forget and miss their registration times and dates, thus finding themselves registering manually on the following semester which is constrained by the course available capacity. Student also relies solely on the existing online systems and services for information related to their academic results, announcement, attendance and timetable updates. This contextual information is all handled by the Student Information Centre via the Online Applications, which at present provides no alerts once the information has been updated. Student need to take their own effort to check with the Information Centre from time to time, or rely on word of mouth from others to know about the updates. Furthermore, students have to keep up-to date with the Multimedia Learning System (MMLS) for new uploaded course materials in order to prepare for their next classes. Basically, it depends on individual student attitude towards his/her education and effort of responsibility to access the Multimedia Learning System.

Moreover, students are expected to complete an online academic evaluation at a specified period of time. This is required to provide feedback to academic on the teaching and learning process for the whole semester. The problem is that students are informed of the evaluation period only upon academics request or announcement, banners around campus and use of online bulletin board available in the Event Management System Even tough the bulletin board is daily 
updated, the announcement is listed based on the announcement owners, i.e. the department, faculty, club or society. Student has to search for bulletins by selecting from the list of that particular day or filter the search for relevant ones.

These problems outline the lack of pervasive applications which provide the right information to the right users, at the right time via the right device as well as improving the acceptability and usability of existing online systems. A system's overall acceptability includes its social acceptability and all practical aspects such as reliability, cost, compatibility and usefulness [1].

Furthermore, with the wide research on the inherent need of student for educational context update surrounding the aspects of mobile computing today, seem to provide tools that can close the traditional barriers of interaction amongst the academics and students. A more collaborative 24/7 environment of such interaction nature is facilitated by the vast availability of mobile devices such as PDAs and mobile phones. Assuming most individual own mobile devices and carry it most of the time, this study aims to propose a mobile application in order to overcome the online systems drawbacks in a university environment by considering the context of use and that the learner should be able to receive personalized information which is valuable to him/her in the given context [2]. An application which examines and reacts to an individual's changing context is defined as a contextaware application [3].

The following sections provide an overview of the framework, describes the architectural design of the proposed application, discuss the implementation and testing results. The final section concludes the study and gives recommendations for future works.

\section{THE FRAMEWORK}

This study adopts event-based approach towards contextaware personalized notification service specially tailored for the stakeholders, i.e. students, academics and the university in mind. The framework deems the stakeholders as event providers, and notification subscribers respectively (Fig. 1). There are four main components - Event Management System, Subscription Management System, Notification Services and Delivery System.

From the student perspective, the SMS notifications of events are provided based on their preferences and current context. In order to receive personalized SMS notifications students must subscribe (free of charge) to events they would prefer to be notified about and set the time they prefer to be notified. Once the student has completed the subscription process, his/her profile is recorded in the event and delivery system.

When event providers create an event such as, an announcement, the application will be triggered to send SMS notification direct to specified subscribers of the event

\section{A. Event Management System}

Event Management System (EMS) receives events from event providers whom are academics or administrator or person-in-charge of several clubs and departments in the university.

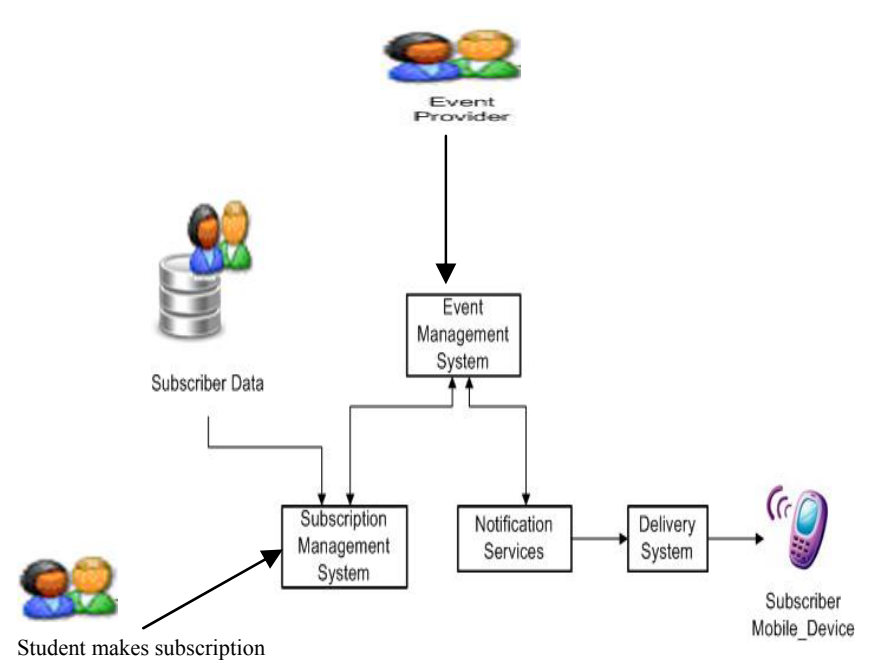

Fig. 1 The framework

\section{B. Subscription Management System}

The Subscription Management System anticipates multiple simultaneous events from the EMS. Together with the Notification Services, EMS is expected to collect, compare or match subscriptions with events to generate immediate notification to be delivered by the delivery system. The subscriber data received through the Subscription Management Application (which is web and mobile based) is separated into three databases - Subscriber, Subscription and Subscriber mobile carrier data. During the Subscription process, Events are collected in the Event Table. The generator evaluates the subscriptions against the events. The notification generator then creates a notification, containing data about the events. The internal notification table accepts notifications written by the generator. It collects all the notifications until they are ready to be distributed by the distributor to the subscriber. The operation of the generator is controlled by the Transact-SQL rules/ Triggers defined for the application as follows:

i. Subscription event rules generate notifications for event-triggered subscriptions.

ii. Subscription scheduled rules generate notifications for scheduled subscriptions.

\section{Delivery System}

The task of the distributor is to format and distribute notifications using a delivery channel, to SMS delivery service. The distributor is in-charge of three main taskmessage formatting, delivery channel and delivery options.

The formatting of the message will allow for professional and user-friendly notification text to be delivered to the mobile device, by using a formatting method such as XSLT (Extensible Stylesheet Transformations). Consideration for formatting the message is important here because different application do not always support the same format of data. For example, the mobile device Short Message Service (SMS) has character constriction per message received and its format differ per mobile device. Where as Delivery Channel are like pipes to Short Message Service (SMS) 
delivery services. That means SMS gateway is used together with its GMS modem to be able to delivery notifications to Mobile Service Providers such as, Digi, Maxis and Celcom. The Mobile Service Providers (MSP) handles the final delivery of the notifications.

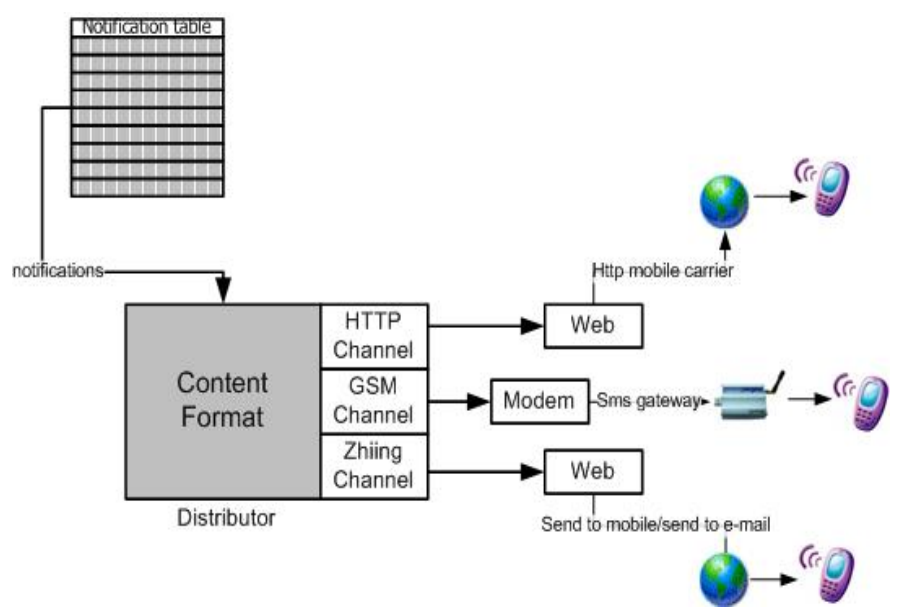

Fig. 2 The delivery system process

There are two Delivery Options which is the bottom line to notification formatting and delivery architecture. First option is digest delivery, which will group multiple notifications to an individual in a single message and the second option is multicast delivery which sends a single notification to multiple subscribers.

\section{Notification Services}

Notification services are added to the existing systems which consists of four types of settings- reminder, general/emergency, compulsory and personalized notification. The reminder setting allows user to specify the time and date they would like the notification service to personally remind them of their schedules. General/Emergency setting is to inform students of any emergency situations and important announcement from various departments. Compulsory setting is applicable to students according to their registered subject. Personalized setting is based on student subscription of preferred events either from the web or mobile based.

\section{PROPOSED APPLICATION}

The proposed application aims to provide a personalized, fast and secure service to the University stakeholders. By implementing the application, student may choose which notifications he/she wants to receive for what period, by subscribing/unsubscribing (personalized). Additionally, the application provides instant notification messages to subscribers (fast) and only student mobile number is used for communication purposes (secure).

This proposed application provides a platform for student to subscribe for SMS notification of the university and personalized them from their mobile phone. To personalize notifications the student will have to subscribe to their choice of events as shown in Fig. 3. Based on Fig. 3, the student or user subscribes memo. This implies that he/she will only receive SMS notifications if any memo is released. The subscriber can also delete or unsubscribe from an event accordingly to stop receiving notifications. Subscribers may also set a period they would like to receive such notifications and they are allowed to subscribe to as many events as they wish.

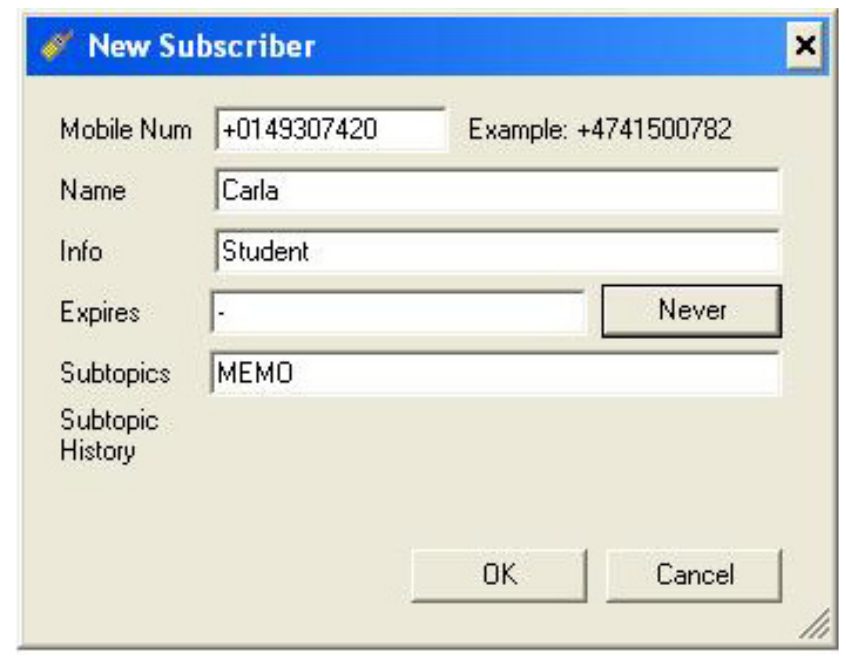

Fig. 3 New subscriber interface

The subscribers and subscription details is stored in the Subscription Management System database. This enables queries and reports to be generated in future in order to provide meaningful information to users, e.g. display list of subscriptions they have made. Subscription details or settings can be modified any time as illustrated in Fig. 4.

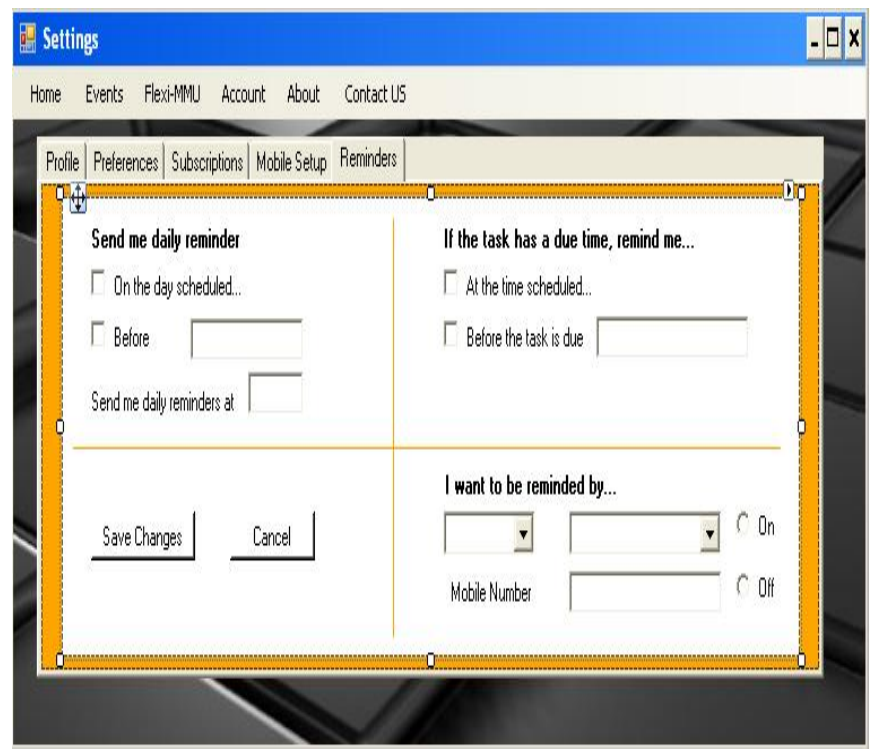

Fig. 4 Subscription details setting interface

For example, Fig. 4 illustrates the interface to set reminders for both Exam Schedule and Subject registration schedule. The reminders will be delivered to subscribers on the day and time specified via his mobile phone. However, in order to receive notifications on any events or reminders, subscribers need to setup their mobile. Fig. 5 illustrates the interface for the mobile setup. 


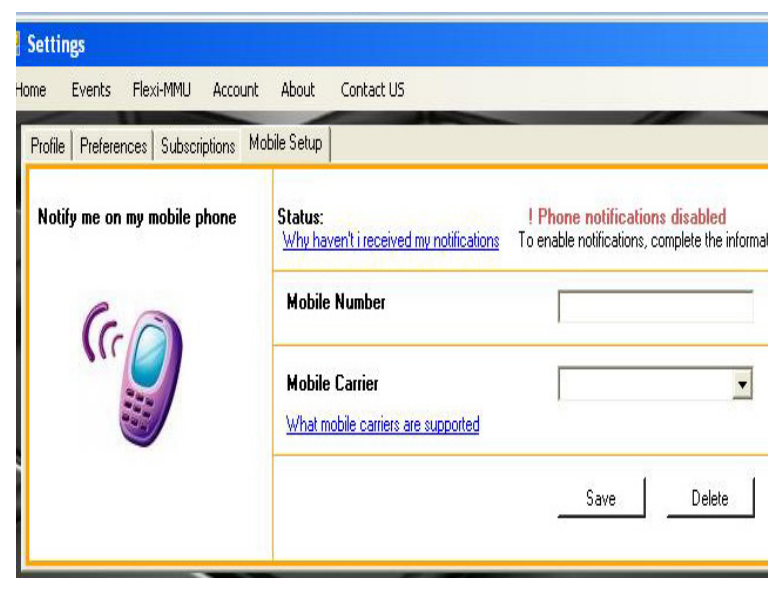

Fig. 5 Mobile setup interface

In general, the Subscription Management System provides the following functions:

i. Creates a new subscriber with the subscription details

ii. Specify the target mobile carrier

iii. Generate notifications from new incoming events

iv. Compare new events to stored subscription information

v. Perform Transact-SQL JOIN statement/ query (Notification Rule) between Events table and Subscription table.

vi. Transact-SQL statement inserts a new record into the Notification table whenever SMS Notification

Services receives a matching event,

vii. Format the notification

viii. Results are the formatted notifications that SMS Notification service must send to subscribers

ix. Specify a delivery protocol to send the notification to the subscriber/s.

Event providers can make add events by utilizing the Event Management System (Fig. 6). For example, if the Security Division wishes to make any special announcements, the details are inserted in EMS. Since this is a general notification, everyone recorded in the database will receive the announcement via their registered mobile. The EMS provides functionalities to add/edit/ delete events, store and view events in the database.

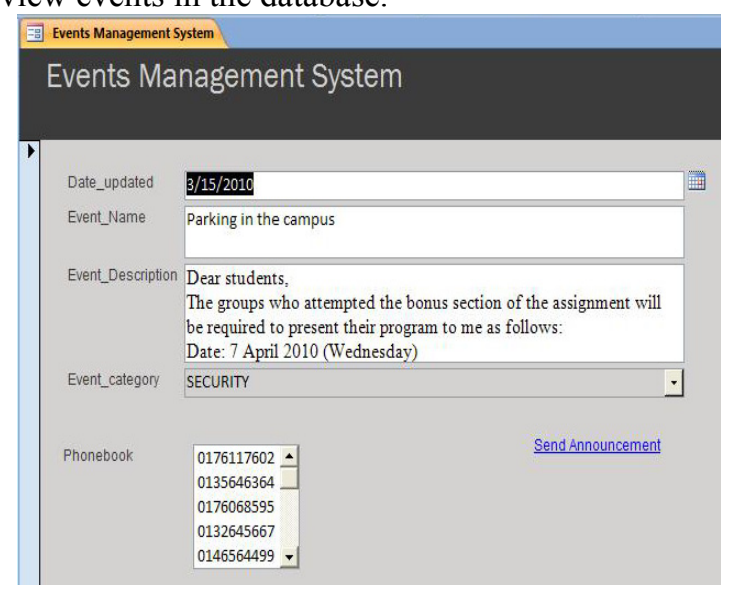

Fig. 6 Interface of Event Management System

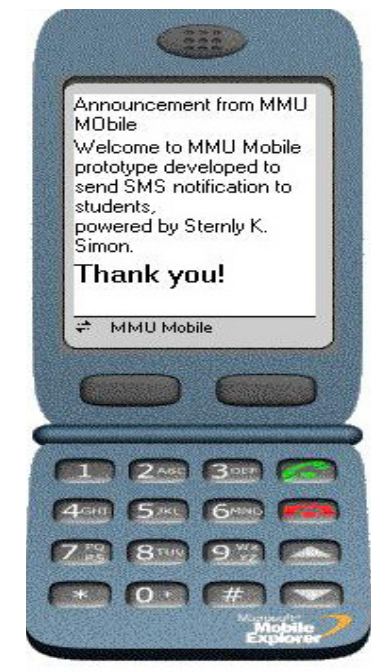

Fig. 7 Sample notification

Fig. 7 illustrates the notification received by the subscriber based on his subscription details. The Delivery Service implements SysMan TellMe V8.04 as the delivery protocol and SMS server.

\section{CONCLUSIONS AND RECOMMENDATIONS}

A web-based portal has been developed to support the implementation of this application. A user acceptance testing was also conducted among potential users of the proposed application. Positive response was received where more than $80 \%$ of respondents are very satisfied with the application. In their opinion the application is very useful and easy to use as well as reduce the problems of using online systems which we have discussed in the earlier section.

We are confident that the proposed application is capable of much more than SMS Notification service. The system can be extended to provide additional functionalities. In addition to allowing the students to subscribe to events from the application, instead a WAP site can be developed in future for the same purpose using Android phones application development platform. Some of the future works of the prototype that can be taken into consideration are in terms of language support, user interface, enhance administrator's capabilities, incorporate other channel modes and incorporate other notification service applications.

The web based portal dedicated to the proposed application could be enhanced to provide more language support such as Malay, Chinese language version besides the current English language version. This will enable information to be displayed in the different language. As a result, this will broaden the usage of the system and interact with more students, even though the official language used in MMU is English.

The user interface of the system can be enhanced to be more attractive, impressive and interactive when this web portal prototype is converted to a real system.

Administrator's task can be further enhanced to include more features to ease maintenance process, and reduce too much manual interaction with the system. For example, in 
the prototype the administrators have to post events and also send the notification to dedicated subscribers.

The prototype is using a GSM delivery channel which presents its own limitations and non-functionalities. The actual real system will be sending bulk SMS to some if not all students and that will require a more sophisticated and dedicate delivery channel like Clickatell bulk SMS gateway. Clickatell is the world's leading SMS messaging provider offering simple and reliable SMS gateway connectivity to 819 networks in 222 countries.

Furthermore, the proposed application is possible to be incorporated with intelligent notification services such as WebSphere Everyplace Access and Microsoft SQL Server 2005 Notification services. This application was unable to employ this software's in its implementation plan and design because they are commercial and requires a much longer time to deploy.

\section{REFERENCES}

[1] Nielsen, J. (2003). Mobile Devices: One generation from useful. Alertbox posting on useit.com: Jakob Nielsen's Website. Retrieved February 14, 2007 from: http://www.useit.com/alertbox/ 20030818.html
[2] O’Malley, C., Vavoula, G., Glew, J.P., Taylor, J., Sharples, M., \& Lefrere, P. (2003). MOBIlearn WP4 - Guidelines for Learning/Teaching/Tutoring in a Mobile Environment. Retrieved February 14, 2007 from: http://www.mobilearn.org/download/ results/guidelines.pdf

[3] Schilit B., Adams, N. \& Want, R. (1994). Context-aware computing applications. Proceedings of the 1st International Workshop on Mobile Computing Systems and Applications, 85-90.Los Alamitos, CA: IEEE.

[4] Bowman, B., Debray, S. K., and Peterson, L. L. Reasoning about naming systems. ACM Trans. Program. Lang. Syst., 15, 5 (Nov. 1993), 795-825.

[5] Ding, W., and Marchionini, G. A Study on Video Browsing Strategies. Technical Report UMIACS-TR-97-40, University of Maryland, College Park, MD, 1997.

[6] Fröhlich, B. and Plate, J. The cubic mouse: a new device for threedimensional iput. In Proceedings of the SIGCHI conference on Human factors in computing systems (CHI '00) (The Hague, The Netherlands, April 1-6, 2000). ACM Press, New York, NY, 2000, 526-531.

[7] Lamport, L. LaTeX User's Guide and Document Reference Manual. Addison-Wesley, Reading, MA, 1986.

[8] Sannella, M. J. Constraint Satisfaction and Debugging for Interactive User Interfaces. Ph.D. Thesis, University of Washington, Seattle, WA, 1994. 